\title{
How simulation can help defining necessary speed sensor-bearing performance for asynchronous motor control
}

\author{
Susanne Blokland ${ }^{1}$ \\ ${ }^{1}$ SKF France, 204 Boulevard Charles de Gaulle, 37540 Saint-Cyr-sur-Loire, Susanne.Blokland@SKF.com
}

\begin{abstract}
This paper presents a method to define the optimum sensor performance to meet customer requirements on asynchronous motor performance parameters such as efficiency or output torque ripple. The method uses simulation: a speed sensor-bearing simulation tool is coupled with an asynchronous motor model of the electromechanical parts as well as the motor control to perform a reduced Design of Experiments study using the extreme outcomes for the sensor as inputs to the motor system model. Subsequently, a mapping of the efficiency or another output parameter of the motor can be realized with respect to the torque and speed range selected. This mapping is checked against the desired minimum performance required by the customer, to finalize the assessment of the sensor-bearing performance with a robustness verification. The method has been applied to a case where two different variants of sensor populations have been tested in a certain given motor and control configuration. The results show that it is possible to predict the necessary sensor-bearing performance in terms of motor performance.
\end{abstract}

induction (asynchronous) motor, modeling, simulation, component, control system

\section{Introduction}

Efficiency is one of the drivers of todays motor design choices, along with the fluctuating price of rare earth. Considering the recent trends in asynchronous motor development for automotive applications, this type of motor can be an answer to both of these challenges. The motor, typically consisting of a squirrel-cage rotor and a wounded stator, is already widely used in industry and its control and inverter need to be adapted to allow torque and speed control, just as for permanent magnet synchronous motors. A challenge in this adaptation is the design of the feedback loop; since automotive applications require a well-performing control, the information necessary to control the motor needs to be accurate and with an high enough resolution. This information consists typically of the mechanical speed of the rotor and its rotation direction. A dual requirement on the speed information makes it difficult to dimension the control signals; during acceleration phases from standstill the speed of the motor shaft needs to be known quite precisely from stand-still on. During continuous op- eration (when constant speed is reached), an update rate of the speed information of once per revolution would be sufficient. Current control strategies include therefore typically two different algorithms for low and high speed[1][2].

For the control signals designating speed and direction, more than one different signal definition is used on the market today. In Table 1, they are summarized.

\begin{tabular}{|c|c|c|c|c|c|}
\hline & $\begin{array}{l}\text { Number } \\
\text { of signals }\end{array}$ & $\begin{array}{l}\text { Signal } \\
\text { definition }\end{array}$ & $\begin{array}{l}\text { Speed } \\
\text { information }\end{array}$ & $\begin{array}{l}\text { Direction } \\
\text { information }\end{array}$ & $\begin{array}{l}\text { Position } \\
\text { information }\end{array}$ \\
\hline PWM & 1 & $\begin{array}{l}\text { Varying duty } \\
\text { cycle }\end{array}$ & From pulses & From duty cycle & Not directly \\
\hline $\mathrm{AB}$ & 2 & Incremental & From pulses & From phase shift & Not directly \\
\hline $\begin{array}{l}\mathrm{A}+ \\
\text { direction }\end{array}$ & 2 & $\begin{array}{l}\text { Incremental and } \\
\text { logic }\end{array}$ & From pulses & From logic state & Not directly \\
\hline $\begin{array}{l}\text { Top-Dead- } \\
\text { Center }\end{array}$ & 1 & Incremental & From pulses & Not directly & $\begin{array}{l}\text { From } \\
\text { missing pulse }\end{array}$ \\
\hline $\mathrm{ABZ}$ & 3 & $\begin{array}{l}\text { Incremental and } \\
\text { index pulse }\end{array}$ & From pulses & From phase shift & Not directly \\
\hline
\end{tabular}

Table 1: Sensor signals for asynchronous motor control

SKF developments for the motor encoder sensorbearing unit, a sensor for asynchronous motor control that is integrated into a bearing, focus on giving speed and direction information. Since the 
Top-Dead-Center sensor is the only sensor not having direct direction information, this output type is not considered in for the motor encoder sensor-bearing unit. This sensor is therefore a pure speed and direction sensor. The sensor output type regarded in this study is the $A B$ type, thus a speed sensor having two incremental outputs with a certain number of pulses per turn, and having a phase shift of 90 electrical degrees typically.

In this paper, a method is proposed for the dimensioning of the control signals (the $\mathrm{A}$ and $\mathrm{B}$ incremental signals) as a function of the algorithm used for speed and direction detection and a selected motor. The method involves a simulation model of the speed and direction detection algorithms and an asynchronous motor model where the following sensor performance parameters are included: number of pulses on the two incremental sensor output signals and duty cycle, phase shift and period accuracy of these signals.

\section{Method}

To be able to assess the motor performance through sensor-bearing performance, modeling on two levels is required. The modeled system is described in paragraph 2.1. In paragraph 2.2, the procedure for the assessment of required sensor performance is explained.

\subsection{System description}

At component level, a model is created that links the sensor-bearing input parameters to its performance. This model is called the Speed SensorBearing Simulation Tool. This tool contains models of all components of sensor-bearings, such as the magnetic target and the Hall-effect or AMR/GMR cells. The variations of the parameters linked to these components are also implemented, such as magnetic field amplitude variations, cell commutation level variations, etc.. In fact, the Speed Sensor-Bearing Simulation Tool combines all SKF knowledge on speed and direction sensor-bearings into one tool.

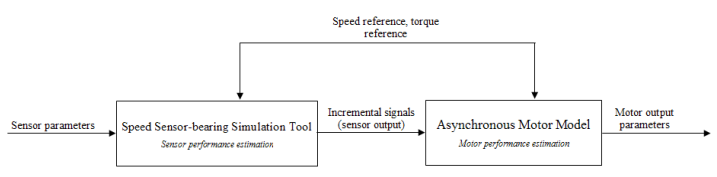

Figure 1: Models

The output of the tool is a measurement of the performance of the sensor-bearings. This measurement can be done using several simulated output parameters such as the duty cycle and period length of the incremental signals, or the phase shift between the two incremental signals. Taking into account these variations of the input parameters, the simulation model is thus able to generate at its output the signals of a full batch of a certain population of sensor-bearings having the same nominal design.
Secondly, a model that links the sensor-bearing performance to the e-motor performance (the Asynchronous Motor Model) is created. The interaction between the two models is shown in Fig.1.

The Asynchronous Motor Model involves, besides a model of the electromechanical part of a squirrel-cage induction motor, a control library in which several algorithms to control the motor are included. In this paper, the focus will be on Direct Field Oriented Control[4]. In this type of control strategy, the torque current component $\left(I_{q}\right)$ and flux current component $\left(I_{d}\right)$ are controlled independently. The DFOC method involves estimation of the rotor flux angle for which information on the rotor speed and stator currents are necessary. As mentioned before, there are several calculation strategies for calculating the speed from the sensor-bearing signals; these algorithms generally include two variations: one for low and one for high speed. The Asynchronous Motor Model has been validated for one type of asynchronous motor, a 600 Watt squirrel-cage induction motor that has been mounted on a test bench. This motor test bench uses a powder brake to provide the load torque. There is an encoder on the motor output shaft which provides a reference speed and position for control. A control model is loaded on the micro controller in a dSpace box to provide the Sevcon motor inverter with the correct PWM inputs.

The interface between the Speed Sensor-Bearing Simulation Tool and the Asynchronous Motor Model is created as follows: the two incremental output signals $A$ and $B$ (evaluated by the tool as a function of rotor angle) are used to calculate the speed error with respect to the base speed using the corresponding speed calculation algorithm (both high and low speed):

$$
V_{\text {error }}=N_{\text {base }}-f\left(A, B, N_{\text {base }}\right)
$$

where $N_{b a s e}$ is the base speed in rpm. This speed error per angular position is entered into a lookup table which is placed inside the motor loop: the shaft speed feedback information is altered by this look-up table using the rotor position. The schematics can be seen in Fig. 2 .

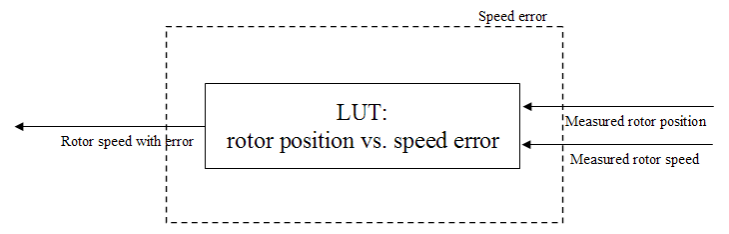

Figure 2: Speed error due to sensor included in feedback loop

\subsection{Procedure}

To determine the best suitable sensor-bearing configuration for which the motor performance 
is optimized a Design of Experiment (DoE) approach is followed using the simulation tools presented in paragraph 2.1. First, populations of sensors are generated using the Speed Sensor Simulation Tool. These populations represent different sensor designs: for instance, magnetic rings with different numbers of pole pairs, different cell references, different electronics, etc.. These different configurations of the sensorbearings will result in sensor-bearings with the same kind of output signals (two incremental signals) but with different performances. A specific design configuration might for instance be able to generate more pulses, but with a period accuracy that is less than another design variant having less pulses per revolution. These different sensor populations will be analysed to include only specific cases: worst cases but also nominal cases. Using only some of the generated sensors will speed up the simulation process when still a lot of different populations exist. With these specific cases defined for each population, the sensor library of the Asynchronous Motor Model can be filled (see Fig.2). In simulation mode, a specific (and variable) torque/speed profile is followed for each of the identified cases to obtain the motor performance mapping on the entire range of operation. These motor mappings are tested for the desired performance. For instance, the efficiency vs. torque and speed is mapped for the worst case sensor performance of a certain population of a certain sensor design configuration. This mapping shows a minimum value of $85 \%$ of efficiency. The customer has expressed the need of having in this certain speed/torque range a minimum efficiency of $87 \%$. The values being fairly close, this sensor configuration will be the subject of a detailed investigation to include the entire population. This will allow for estimation of the amount of sensors in a population that will be exceeding the minimum requirement. If the population limits yield sufficient performance, the sensor design configuration is acceptable for reaching the efficiency on the selected customer motor and control algorithm.

\section{Simulation results}

The simulation results are obtained following the method described above. First, simulations of the sensor-bearing are performed. Their results can be found in paragraph 3.1. Then, these simulations are added to the electric motor system; the results of these simulations can be found in paragraph 3.2.

\subsection{Sensor simulation}

The first step for simulation is to model the sensor populations for each considered design. The requirements for this speed sensor are:

- Minimum 64 pulses per signal per revolution

- Number of pulses must be a power of 2
- Performance such that the motor efficiency does not drop below $87 \%$

In this example, the choice is made to create only two populations using the same design configuration for simplicity. Simulation results are shown for sensor-bearings having two different numbers of pulses (64 and 128 pulses per signal per turn) using the results of 1,000 simulated sensors per population in Fig. 3 and 4.

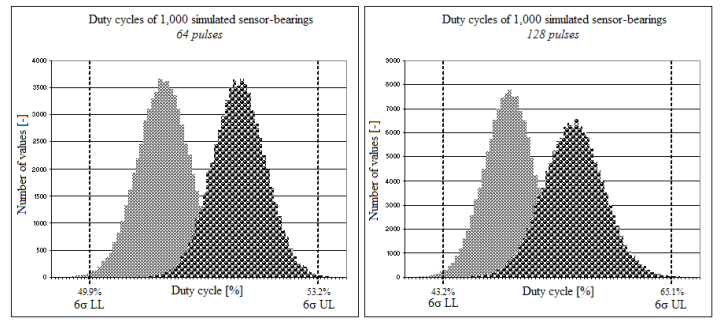

Figure 3: Duty cycle simulation results

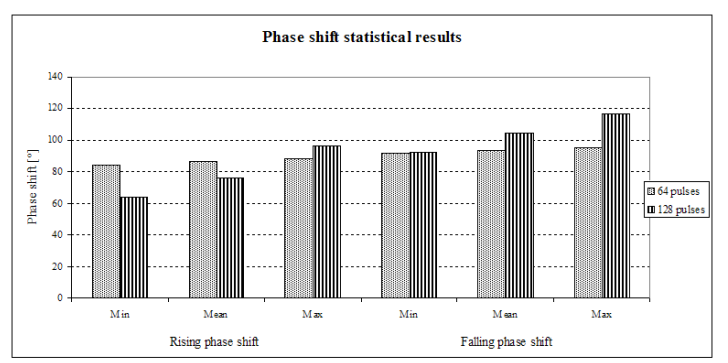

Figure 4: Phase shift simulation results

It can be observed that the performances for the sensor-bearing having 128 pulses are less accurate than for 64 pulses, since the phase shift and duty cycle have larger standard deviation. This is inherent to the specified design. Typically, these results reflect a design trade-off: more pulses with less accuracy or less pulses with better accuracy. It all depends on the motor and control strategy now to define which sensor configuration suits better.

The cases that are chosen for simulation inside the e-motor model for both populations are the 6 Sigma upper and lower limits for the duty cycle as indicated in Fig.3.

\subsection{E-motor system simulation}

The chosen control algorithm is a control algorithm using two strategies, depending on the speed of the shaft[3].

- Low speed: measures time in [s] between rising edges on encoder signals

- High speed: measures number of edges (rising and falling) in fixed time window $\left(t_{h i g h}\right.$ in [s]) on encoder signals 
The high speed algorithm is always active to detect the speed. When the speed is lower than a certain switching speed, $\omega_{\text {switch }}$, the low speed algorithm output is used for control. The equation for the switching speed is:

$$
\omega_{\text {switch }}=\frac{60 P}{t_{\text {high }} N b_{\text {pulses }}}[\mathrm{rpm}]
$$

where $P$ is the minimum number of pulses in the fixed time window to use the speed information calculated by the high speed algorithm and $N b_{\text {pulses }}$ the number of pulses per revolution.

The speed errors are thus dependant on the speed itself. In Fig.5 examples of speed errors at high and low speed are shown for the sensorbearing with minimum value for the duty cycle (64 pulses).

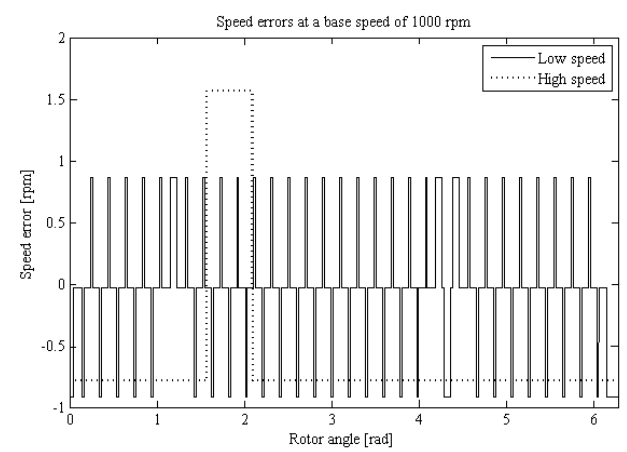

Figure 5: Speed errors of minimum duty cycle sensorbearing simulation

For different speed and torques, the motor model is run. Inside the speed feedback loop, the speed error caused by the two sensors (with 64 and 128 pulses per turn) is added. The simulation results have been plotted for the following output parameters: the efficiency and the torque ripple standard deviation (a measure of the torque quality). Those two parameters are quite important for motor manufacturers: the efficiency must be high to minimize the energy that has to be put in the motor to make it run. The torque ripple standard deviation is measured over time and gives thus an indication of the variation of the torque over time. This value needs to be as close to zero as possible, since this means less ripple is present and as a consequence, there are as less as possible unwanted vibrations in the car.

In Fig. 6 the efficiency results are shown for both sensor configurations (64 and 128 pulses) having minimum duty cycle measured over the sensor population. The results for sensors having minimum duty cycle is shown here, since at first, worst cases are regarded as explained in paragraph 2.2. It can be seen that the efficiency diagrams are similar in shape, but that the 128 pulses solution always gives higher efficiency, between 0 and $15 \%$ more (especially at high torque). Similar diagrams have been obtained during the simulations, again with minimum duty cycle, showing the standard deviation of the torque. The results can be seen in Fig.7.
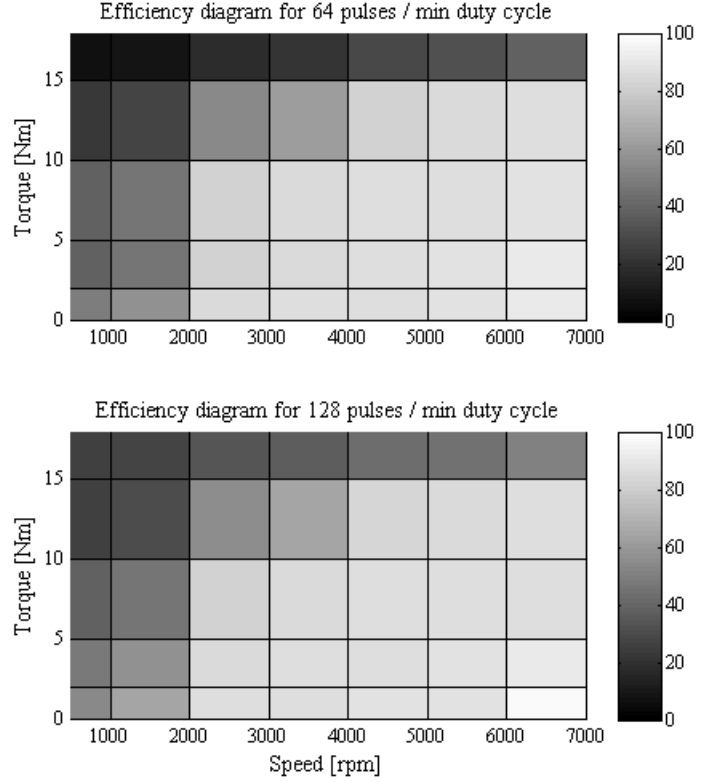

Figure 6: Efficiency diagrams

The torque standard deviation at low speeds and high torque is worse for the 128-pulses sensor, but this sensor gives lower torque standard deviation at high speeds and low torque except between 6000 and $7000 \mathrm{rpm}$ and $0-2 \mathrm{Nm}$ of torque. With the results presented in Fig.6 and 7, it can be concluded that for the minimum duty cycle, the significant differences in the efficiency and torque standard deviation mappings between the two sensor configurations are mainly present at low speeds and high torque.
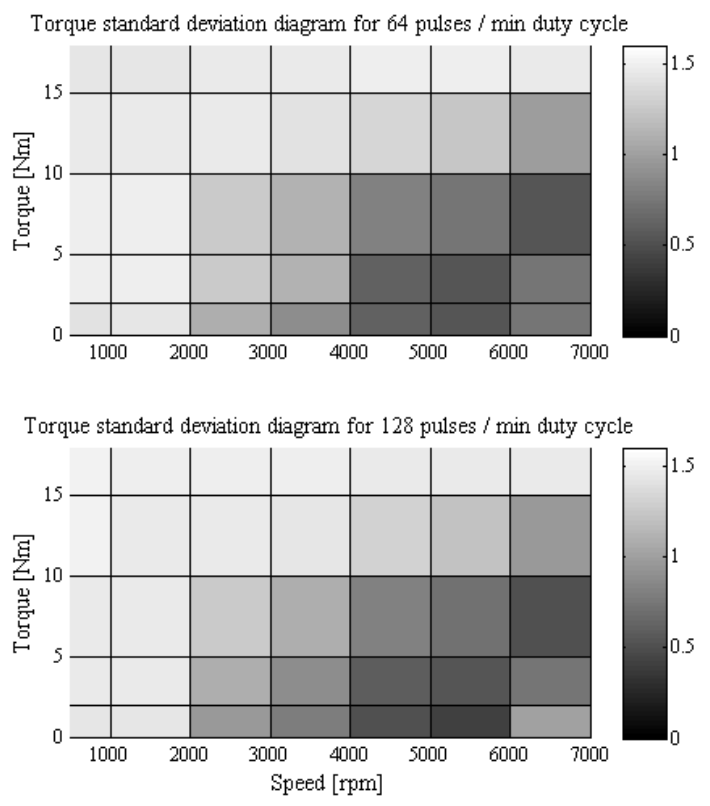

Figure 7: Torque standard deviation diagrams

With the example of a minimum efficiency of $87 \%$ both sensors behave similarly, but the 128 pulses sensor has slightly less torque ripple in the 
concerned efficiency region and light be thus the better choice in this case. Linking these system simulation results to the sensor simulation results presented in paragraph 3.1, means that even though the measured accuracy of the 128 pulses sensor is worse than that of the 64 pulses sensor (its phase shift and duty cycle have larger deviations) its performance in the motor is slightly better. This can be explained by the higher number of pulses, which gives information at a higher rate about the actual speed and thus the speed is determined more often. So for the example presented in this paper, only looking at the sensor performance would have concluded that the sensor having 64 pulses is more accurate. But including the speed calculation algorithms and the motor, it can be seen that the benefit of having 128 pulses is higher than the loss of accuracy on the signals, since the efficiency is higher.

Since in this example the analysis has been done with one sensor simulation only (minimum duty cycle), for a complete assessment, other extreme points should be considered to confirm the results.

\section{Conclusions}

From the simulations, it can be concluded that it is possible to quantify the influence of several design parameters of a sensor-bearing population in order to reach the required asynchronous motor performance. A limited Design of Experiment approach is used to identify the sensor populations responding favourably to the application's required motor performances, and a full investigation of a selected sensor configuration can be done to optimize the sensor-bearing performances in order to obtain robust performance of the complete motor system.

\section{Recommendations}

Simulations can be checked by performing hardware-in-the-loop analysis using the test bench presented in section 2 . In the same way as in simulation, the simulated sensor signals denoting different performances can be integrated in a sensor library, and the calculated speed from the reference encoder on the motor output shaft can be altered to represent specific sensor cases. The e-motor efficiency maps can now be created using the real customer motor instead of a model, and will determine whether the sensor-bearing performance is indeed sufficient as found in simulation.

\section{References}

[1] I Miyashita and Y Ohmori, A new speed observer for an induction motor using the speed estimation technique, Journal of Power sources, ISSN 0378-7753, 162(2006), 913-919.
[2] R. Petrella and others, An embedded system for position and speed measurement adopting incremental encoders, IEEE Transactions on Industry applications, Vol 44, No. 5, Sep/Oct 2008.

[3] J.J. Incze and others, Modeling and simulation of an incremental encoder used in electrical drives, 10th international symposium of Hungarian Researchers on Computational Intelligence and Informatics, November 12-14, 2009.

[4] Mircea Popescu, Induction motor modelling for vector control purposes, Helsinki University of Technology Department of Electrical and Communications Engineering Laboratory of Electromechanics, Espoo 2000.

\section{Authors}

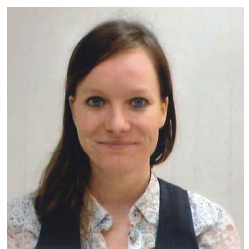

Susanne Blokland has been working in the Automotive Development Center - Sensor Integration belonging to SKF Automotive since February 2010. She is responsible for capturing the voice of the customer and translating it into technical requirements for sensorbearings mainly used for electric motor control. She holds a Bachelor degree in Mechanical Engineering and a Master of Science degree in Control Engineering from the Delft University of Technology in the Netherlands. 